\title{
Du terrain au Tour du monde : la fabrique du lointain
}

From the field to Le Tour du monde: manufacturing remote images

\section{Victoire Lallouette}

\section{OpenEdition Journals}

Édition électronique

URL : http://journals.openedition.org/cel/571

DOI : $10.4000 /$ cel. 571

ISSN : 2262-208X

Éditeur

École du Louvre

\section{Référence électronique}

Victoire Lallouette, «Du terrain au Tour du monde : la fabrique du lointain », Les Cahiers de l'École du Louvre [En ligne], 10 | 2017, mis en ligne le 03 mai 2017, consulté le 17 septembre 2019. URL : http:// journals.openedition.org/cel/571 ; DOI : 10.4000/cel.571

Ce document a été généré automatiquement le 17 septembre 2019.

\section{(c) $($ i) $(9)$}

Les Cahiers de l'École du Louvre sont mis à disposition selon les termes de la licence Creative Commons Attribution - Pas d'Utilisation Commerciale - Pas de Modification 4.0 International. 


\title{
Du terrain au Tour du monde : la fabrique du lointain
}

From the field to Le Tour du monde: manufacturing remote images

\author{
Victoire Lallouette
}

1 À la fin du XIX $x^{e}$ siècle, les politiques impériales des pays européens mènent à l'annexion de nombreux territoires du Pacifique. Même si elle reste en très grande partie inconnue des Occidentaux car très peu explorée - les terres intérieures ne seront foulées par les Blancs qu'à partir des années 1950 -, la Nouvelle-Guinée ne fait pas exception : l'ouest de l'île passe aux mains des Pays-Bas dès le début du siècle alors que la moitié est est partagée entre l'Allemagne au nord et le Royaume-Uni au sud. Si la France est principalement présente en Polynésie, la Mélanésie n'échappe pas pour autant aux missionnaires français. Ainsi, une petite confrérie de pères d'Issoudun s'installe en face de Port-Moresby, centre de la récente annexion britannique. En 1895, ils reçoivent la visite d'un explorateur français, le marquis de Cacqueray de Lorme. Ce dernier réalise quelques photographies qui nous sont parvenues sous la forme d'un ensemble de neuf plaques de projection. Actuellement conservées au musée du quai Branly - Jacques Chirac à Paris, elles proviennent du laboratoire d'anthropologie du Muséum d'histoire naturelle au sein duquel elles sont arrivées en 1896, comme l'indique l'étiquette apposée sur chacune d'entre elles. Hormis le nom de leur auteur, aucune information n'accompagne ces images que nous allons tenter d'analyser.

Notre étude ne serait pas complète sans l'inclusion d'un matériau supplémentaire : la presse illustrée. C'est dans la comparaison entre le voyage vécu et le récit rapporté que se loge l'intérêt que nous portons à la figure du marquis de Cacqueray de Lorme. Après avoir reconstitué le contexte historique de production de ces images photographiques, il s'agira d'analyser en détail le contenu des plaques de projection et d'expliciter leur utilisation. Ceci nous permettra d'interroger les rapports entre ces objets et les récits postérieurs à l'expédition du marquis, rapportés dans les bulletins des sociétés savantes et publiés dans la presse illustrée. 


\section{Le Sacré-Cœur de Jésus : l'installation française en Nouvelle-Guinée}

2 L'installation missionnaire en Nouvelle-Guinée est d'abord anglaise avant d'être française : les premiers missionnaires britanniques s'installent au sud-est de l'île dans les années 1875. Une dizaine d'années plus tard, ils seront rejoints, à leur grand désarroi, par des frères catholiques français. Comme dans de nombreuses îles du Pacifique, la conquête des âmes est le théâtre d'oppositions voire d'affrontements entre les deux Églises chrétiennes. Mais si les missionnaires nous intéressent ici, c'est avant tout pour le rôle de médiateurs qu'ils jouent entre les populations de Nouvelle-Guinée et les explorateurs occidentaux.

Très Révérend Père, Depuis plusieurs années, le Vicariat de la Nouvelle-Guinée est vacant, faute d'une communauté religieuse qui veuille s'en charger. Le Saint-Siège, qui porte le plus grand intérêt à cette importante contrée où n'existe aucune Mission catholique, tandis que plus d'un ministre protestant y répand l'erreur [...] verrait avec un très grand plaisir les Missionnaires du Sacré-Cœur se charger d'évangéliser ce vaste champ ${ }^{1}$.

3 Ainsi commence l'histoire de la mission catholique française en Nouvelle-Guinée. Le destinataire de cette lettre envoyée depuis Rome est Jules Chevalier ${ }^{2}$, fondateur en 1855 de la Société des Missionnaires du Sacré-Cœur de Jésus qui compte une soixantaine de membres en 1881. La proposition acceptée, la Société devient alors responsable du vicariat apostolique de Mélanésie et de Micronésie. Six hommes sont alors envoyés pour établir les bases de la mission, quatre débarquent en Nouvelle-Bretagne, une île située au nord-est de la Nouvelle-Guinée, en septembre 1882. Deux ans plus tard, les catholiques décident d'aller s'établir sur la grande île de Nouvelle-Guinée, qui avait été visée en premier lieu. Après une transition par Thursday Island, ils débarquent à Yule Island le 30 juin 1885, où ils rencontrent les Roros pour la première fois. À la différence du naturaliste italien Luigi d'Albertis qui, en 1875, avait vu dans Yule Island une terre intéressante, les missionnaires s'y établissent faute de mieux, Port Moresby étant chasse gardée des protestants de la Société missionnaire de Londres. Peu d'images subsistent de l'installation de la mission catholique sur la côte sud-est ${ }^{3}$; bien que restreinte, la production de Cacqueray de Lorme devient alors une source intéressante.

\section{Un marquis explorateur}

Auguste Honoré Charles naît le 22 décembre 1862 dans l'Aisne ; il est l'aîné d'une fratrie de quatre garçons et sera le propriétaire du château de Boismorin, vers Vaillysur-Aisne ${ }^{5}$. À trente-deux ans, il effectue une expédition d'au moins six mois en Nouvelle-Guinée britannique : il débarque sur Yule Island le 12 décembre $1894^{6}$, reçu par les missionnaires catholiques du Sacré-Cœur d'Issoudun. Il visite la zone du SaintJoseph, un des longs fleuves néo-guinéens. D'après les comptes-rendus de la Société de géographie, son séjour prend fin de manière brusque et involontaire ${ }^{7}$; quatre ans plus tard, en 1900, un article blâme ouvertement la population de l'île pour cet arrêt soudain : "Ce voyage, extrêmement intéressant, a malheureusement été interrompu par le mauvais vouloir des indigènes ${ }^{8} »$. Outre quelques publications du récit de son expédition, parfois illustrées et sur lesquelles nous reviendrons ultérieurement, nous perdons sa trace jusqu'à la Première Guerre mondiale. Cacqueray de Lorme meurt, 
«tué à l'ennemi", le 25 septembre 1915 à Saint-Thomas-en-Argonne, en Marne ${ }^{9}$. Le château sera anéanti par les bombardements.

5 L'explorateur français arrive chez les missionnaires à un moment clé de l'installation catholique en Nouvelle-Guinée: les Pères du Sacré-Cœur initient de grandes explorations à l'intérieur des terres dans le but d'établir de nouvelles stations. Cette stratégie d'occupation du territoire fait pendant à celle de leurs confrères britanniques protestants qui se déploient depuis une dizaine d'années le long des rivages sud-est. Le père André Jullien ${ }^{10}$ sera l'un des premiers explorateurs catholiques des terres intérieures. Figure emblématique de la mission catholique, il arrive à Yule en février 1894, accompagné de cinq jeunes scolastiques dont le Belge Victor de Rijcke ${ }^{11}$. Jullien et Rijcke effectueront, en 1896, la première grande expédition catholique dans les montagnes. Tous deux sont portraiturés par Cacqueray de Lorme en compagnie d'un jeune garçon et d'un adulte néo-guinéens (fig. 1).

Fig. 1
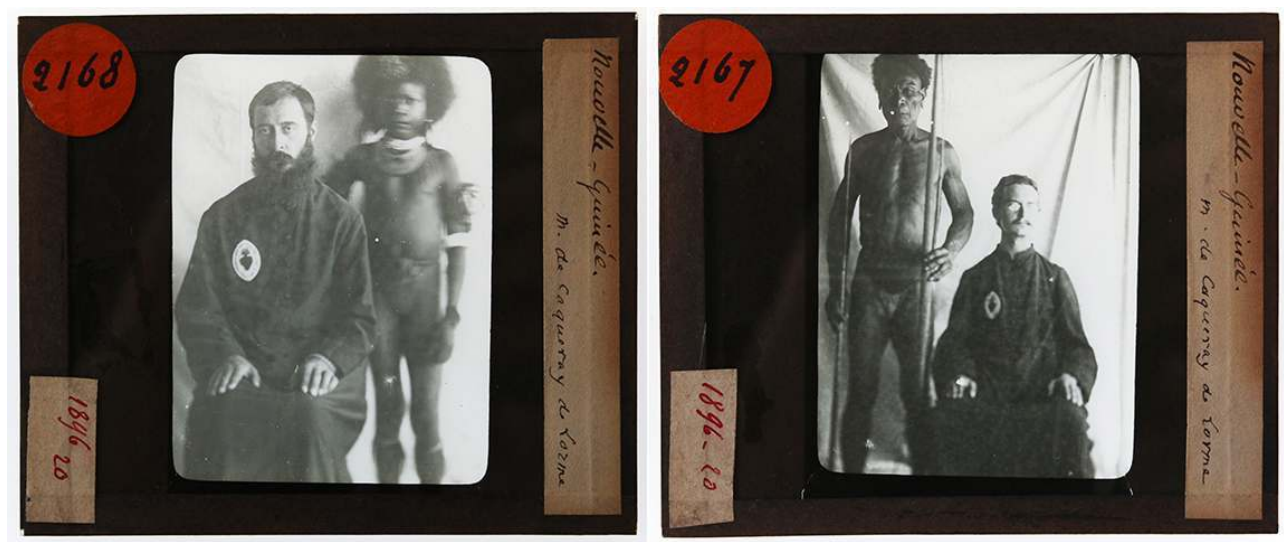

Charles Auguste de Cacqueray de Lorme, Nouvelle-Guinée, PV0045257 et PV0045256, plaques provenant de la collection de plaques de projection du Musée de l'Homme @ musée du quai Branly Jacques Chirac, dist. RMN-Grand Palais / image musée du quai Branly - Jacques Chirac. Le père Jullien est à gauche, le père Rijcke à droite. Toutes les plaques de projection mesurent $8,5 \times 10$ centimètres

\section{Conférences publiques et visions projetées : dans la lignée de l'anthropologie physique}

Le tout premier écho du voyage du marquis est daté de l'année 1896. «À la Société de Géographie - lit-on dans La Presse - 184 boulevard Saint-Germain, vendredi prochain 19 juin, à huit heures et demie du soir, M. le comte de Cacqueray de Lorme, fera une conférence sur la Nouvelle-Guinée anglaise ${ }^{12} »$. La date de cette première conférence coïncide avec l'entrée des neuf plaques de projection au sein des collections du laboratoire d'anthropologie du Muséum national d'histoire naturelle. Il est donc tentant de penser que ces photographies ont été projetées au cours de cette conférence du 19 juin 1896: la Société de géographie est alors une fervente défenseuse de l'instruction populaire et de l'enseignement par l'image. La première conférence illustrée y a lieu dès 1875, grâce au savoir-faire développé par la firme Molteni, principale représentante de l'« enseignement par les projections lumineuses ${ }^{13}$ ». En 1878, un nouveau bâtiment est inauguré pour la Société : la salle de conférences compte 
alors quatre cent cinquante places et devient «l'une des [salles les] plus modernes de Paris $^{14}$ ». Le procédé des projections lumineuses est à son apogée à la fin du XIX siècle, et à partir de 1890 il est même possible de se procurer directement dans le commerce des plaques de verre gélatino-argentiques laissant aux amateurs la possibilité de préparer leurs propres diapositives ${ }^{15}$. L'universalité et l'immédiateté que l'on associe alors à la compréhension des images photographiques font écho aux conditions d'accès à ces conférences libres et ouvertes à tous promouvant l'instruction populaire.

7 Le 20 juin 1901, Cacqueray de Lorme donne une conférence à la Société d'anthropologie de Paris au cours de laquelle il présente des photographies et objets de la NouvelleGuinée anglaise pour appuyer sa démonstration sur les origines des populations néoguinéennes :

Parmi les types qui figurent sur les photographies du marquis de Cacqueray, il s'en trouve un qui présente un profil comparé par l'auteur au profil des Américains du Nord. [...] L'hypothèse des croisements dans cette grande île est d'autant plus plausible que le marquis de Cacqueray vient de nous montrer des portraits d'insulaires possesseurs d'une énorme chevelure ébouriffée [.... ${ }^{16}$.

L'anthropologie de la fin du XIX siècle s'attache à l'étude des origines des populations et des différences entre les «races"; cette conférence de 1901 repose principalement sur des constats d'anthropologie physique qui mènent le marquis jusqu'en Amérique ${ }^{17}$. La photographie devient le support de ce débat :

M. Zaborowski. - [...] Les photographies des tribus de l'extérieur montrent le type Papoua. Celles des tribus de l'intérieur montrent des individus différents, plus musclés, plus robustes, difficiles à comparer aux Indiens de l'Amérique du Nord, car les cheveux sont plats ${ }^{18}$.

Les rares références aux photographies laissent à penser que d'autres images que les plaques de projection étudiées ont été montrées lors de cette conférence. Deux hypothèses coexistent : plusieurs photographies du marquis nous restent inconnues et/ ou l'explorateur a utilisé des photographies dont il n'était pas l'auteur pour soutenir le propos développé dans sa conférence. Il n'était pas rare que certains scientifiques ou explorateurs n'ayant pu réaliser leurs propres photographies procèdent à des emprunts ponctuels pour illustrer leur propos.

8 Quoi qu'il en soit, qu'elles aient été projetées en 1896, en 1901, au Muséum, ou plus tard au Musée de l'Homme où elles furent conservées avant d'entrer au musée du quai Branly - Jacques Chirac $^{19}$, ces neuf plaques de verre servent la démonstration par l'image et trouvent leur raison d'être dans le principe de la conférence. On a vu que Cacqueray ne souhaite pas uniquement témoigner de son voyage, mais qu'il entend développer des théories scientifiques fondées sur ses observations. L'étude comparative de ces neuf plaques de projection nous permet d'extraire quelques points d'intérêt que le marquis jugea opportun de fixer. 
Fig. 2
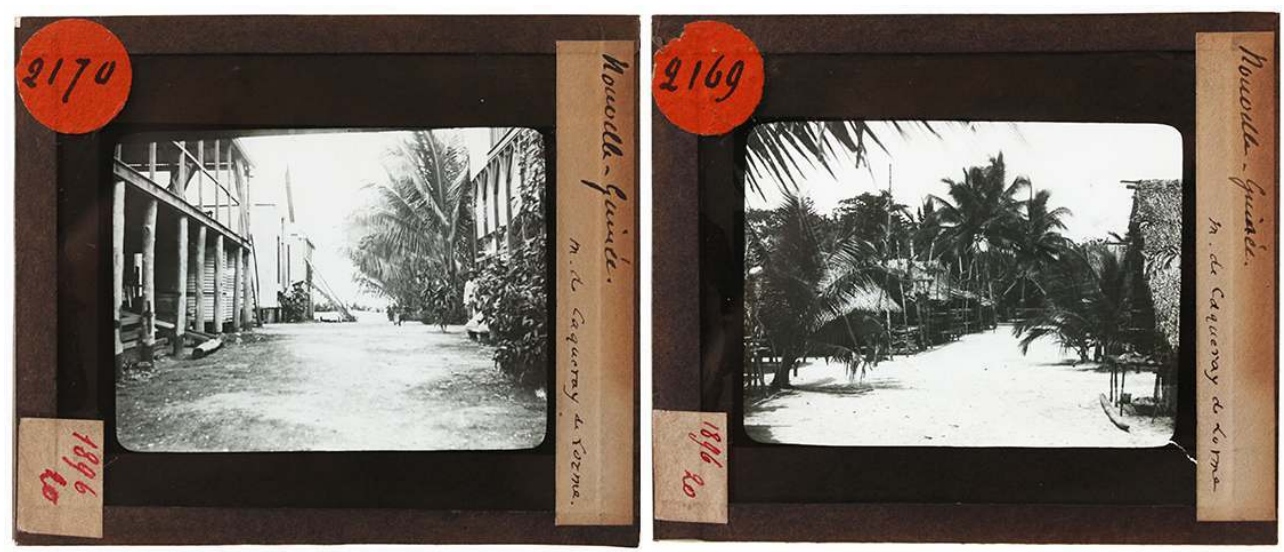

Charles Auguste de Cacqueray de Lorme, Nouvelle-Guinée, PV0045260 et PV0045258, plaques provenant de la collection de plaques de projection du Musée de l'Homme @ musée du quai Branly Jacques Chirac, dist. RMN-Grand Palais / image musée du quai Branly - Jacques Chirac.

\section{La photographie comme outil comparatiste}

Ainsi, les plaques peuvent s'organiser par microséries jouant de la proximité thématique et du comparatisme culturel. À la vue d'une rue bordée de constructions missionnaires répond celle d'un village néo-guinéen aux constructions sur pilotis (fig. 2). Dans la même dynamique, un portrait collectif de Néo-Guinéens assis sur une plate-forme fonctionne en miroir avec un portrait des missionnaires catholiques dans une embarcation. Un portrait collectif rassemble quant à lui missionnaires et insulaires dans une composition classique et symétrique (fig. 3) : comme un trait d'union entre les deux portraits précédents, cette vue réunit trois missionnaires catholiques au centre, tout de blanc vêtus, et dix adultes et enfants néo-guinéens, torses nus. Ce portrait a pu être pris sur l'île de Yule dans le village de Tsiria dont le père Jullien, debout à droite sur la photographie, prend la tête à partir de 1895. La présence d'insulaires n'ayant pas adopté la mode vestimentaire occidentale marque la différence entre ceux qui se réclament d'une civilisation supérieure et les populations alors considérées comme sauvages ; cette vue met en présence l'œuvre " civilisatrice » des missions catholiques et la supposée nature sauvage et exotique, représentée tout autant par les grandes feuilles de palmiers qui occupent les coins supérieurs de l'image que par la nudité des insulaires. 
Fig. 3

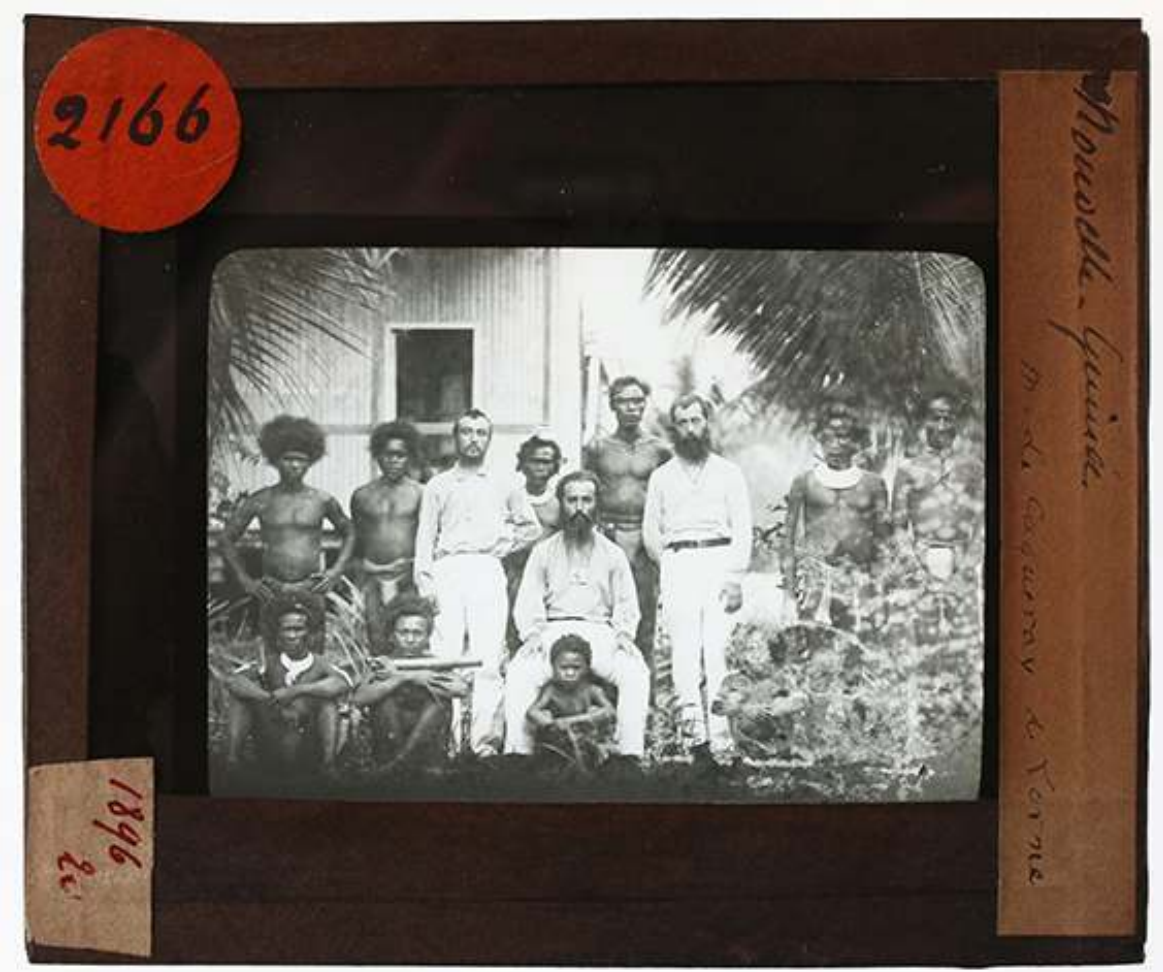

Charles Auguste de Cacqueray de Lorme, Nouvelle-Guinée, PV0045255, plaque provenant de la collection de plaques de projection du Musée de l'Homme @ musée du quai Branly - Jacques Chirac, dist. RMN-Grand Palais / image musée du quai Branly - Jacques Chirac

10 Cette construction se fonde sur l'appréciation d'un contraste entre les Français et les Néo-Guinéens; elle est à nouveau mobilisée dans les deux portraits des pères André Jullien et Victor de Rijcke (voir fig. 1). Cette mise en scène, peu courante dans les portraits de missionnaires en Papouasie, se retrouve dans les publications de la Société des Missionnaires du Sacré-Cœur et en particulier dans un album publié en $1898^{20}$. Au milieu des images de Yule Island, un portrait du « Frère Mariano, l'un des plus anciens missionnaires » reprend une composition similaire : le frère est assis au premier plan, un jeune garçon néo-guinéen se tient debout derrière lui. Le léger flou de l'arrière-plan végétal permet aux silhouettes de se détacher du fond sans pour autant égaler l'effet dramatique des portraits du marquis de Cacqueray de Lorme. Un drap blanc est tendu en arrière-plan : André Jullien et Victor de Rijcke posent tous deux assis, les mains sur les cuisses; derrière eux se tiennent respectivement un jeune garçon et un homme adulte. À nouveau, la nudité de ces derniers contraste avec les soutanes noires des pères sur lesquelles on remarque, dans un cartouche ovale et blanc, l'insigne de la mission catholique. Le clair-obscur du portrait de Victor de Rijcke est saisissant : les plis du drap donnent à la scène un air théâtral. L'homme debout, dont la force physique est exaltée par la lumière tangente, fixe l'appareil. La lance qu'il tient dans la main gauche accentue son air menaçant et sa posture hiératique le rapproche du kouros grec. À l'inverse, le regard du prêtre se perd hors du cadre: son corps assis et ses épaules tombantes renvoient une impression de calme à laquelle le regardeur associe aisément la sérénité religieuse. Ces portraits sont sans aucun doute les plus originaux et les plus surprenants qu'il nous ait été donné de voir dans l'imagerie missionnaire en Nouvelle- 
Guinée ; ils incarnent les préjugés raciaux et culturels qui apparaissent en Europe dès le milieu du XIX ${ }^{e}$ siècle, associant brutalité et sauvagerie aux populations néo-guinéennes.

\section{Du compte-rendu scientifique...}

11 Ces neuf plaques témoignent de l'installation catholique autant qu'elles la mettent en scène en évoquant les avancées de la «mission civilisatrice». Cacqueray partage les préjugés raciaux de la majorité des savants de son temps, pour preuve la retranscription de la séance du 20 juin 1901 à la Société d'anthropologie de Paris :

M. d'Echerac - Nous sommes sévères pour les Papouas, sont-ils plus intelligents que les nègres? $\mathrm{M}$. de Cacqueray répond qu'ils sont susceptibles de comprendre et de soutenir une conversation, pas longtemps cependant à cause de leur esprit volage, il n'a pas soutenu une conversation avec eux ${ }^{21}$.

La réponse du marquis trahit la condescendance de l'explorateur français en terre lointaine, mais elle symbolise également le manque de sérieux de l'étude de terrain bien que l'expression soit anachronique. Il promeut une vision ancrée dans le terreau racialiste de la fin du siècle ; en 1872, Jules Girard, futur secrétaire adjoint de la Société de Géographie de Paris, décrit ainsi les populations papoues :

Leur face est plate ; les sourcils sont accentués, le nez large et écrasé, la couleur de la peau est noire, sale, brune et quelquefois jaune [...]. Leur aspect hideux et leur petite taille ont plusieurs fois laissé supposer qu'ils appartenaient à une variété de nègres orientaux $[. . .]^{22}$.

12 En parallèle des comptes-rendus officiels des communications aux Sociétés de Géographie et d'Anthropologie en 1896 et 1901, trois courts articles sont publiés dans Le journal des voyages et des aventures de terre et de $\mathrm{mer}^{23}$ et à deux occasions dans la Revue de Géographie ${ }^{24}$ :

[Le] climat est malsain [...]. La végétation y est luxuriante, mais la faune peu variée. Les Papous, qui l'habitent, vivent à l'état complètement sauvage. Ce sont des hommes bien musclés, [...] mais d'une intelligence peu éveillée et d'une nature indolente. [...] M. de Cacqueray pense qu'on aura beaucoup de peine à les civiliser ${ }^{25}$.

Ces publications reprennent les grandes lignes des comptes-rendus officiels qu'elles reformulent très légèrement :

Leur intelligence est peu vive, leur nature très paresseuse. [...] une grande partie des tribus sont anthropophages, mous pour le travail, ardents pour la guerre. Au milieu de ces indigènes on peut se croire revenu à l'âge de pierre ${ }^{26}$.

\section{... au récit d'aventures}

Mais un autre type d'article apparaît en 1900 dans La Science Française : revue populaire illustrée, un hebdomadaire publié pour la première fois en 1890. Gaston Bertrand, collaborateur régulier de la revue, rédige les « Pérégrinations d'un compatriote dans la Papouasie inconnue ${ }^{27} »$. Cet article de plus d'une page est considérablement détaillé par comparaison avec les précédents ; l'auteur mentionne à diverses reprises les «notes " de Cacqueray de Lorme auxquelles il a certainement eu accès ${ }^{28}$. Gaston Bertrand inscrit le marquis dans la lignée de Luigi d'Albertis - explorateur et naturaliste italien qui fut le premier Européen à relever le cours du Fly en 1875 - tout en moquant les observations de ce dernier sur le Saint-Joseph : 
[D'Albertis] se borna à relever seulement les côtes, si bien qu'il prit les bouches $\mathrm{du}$ Saint-Joseph pour de simples criques et ne parut même pas soupçonner l'existence dudit fleuve, lequel a été découvert en 1891 par les Pères de la mission [...] ${ }^{29}$.

Dans les mots de Gaston Bertrand, Cacqueray dépasse ainsi l'œuvre du naturaliste italien qui fait alors référence dans l'exploration de la Nouvelle-Guinée. Le marquis est présenté comme un aventurier téméraire « qui n'a pas froid aux yeux » :

M. de Cacquerey [sic] est retourné au Saint-Joseph qu'il a remonté pendant 25 kilomètres environ, sans se laisser arrêter par les rideaux d'arbres à trouer, les amoncellements de débris de végétaux à enjamber, non plus que les crocodiles à affronter et qui sont plus que nombreux dans cette partie du fleuve ${ }^{30}$.

L'article fait la part belle à la géographie et aux sciences naturelles : le fleuve du SaintJoseph, la flore et la faune sont les principaux points développés. L'auteur évoque néanmoins la cause de l'interruption de l'exploration du marquis, «l'animosité des naturels ${ }^{31} »$, " soumis aux népôs ou sorciers, [...] ennemis-nés des blancs, [qui] les incitent à toutes sortes de tours pendables envers les fils de Japhet ${ }^{32}$. » C'est dans ce contexte que l'article reprend l'anecdote qui sera contée un an plus tard à la Société d'anthropologie sur les rituels impliquant mutilations et pratiques cannibales auxquels l'explorateur français aurait assisté.

Quatre années plus tard, Le Journal des Voyages et des Aventures de terre et de mer présente "Les Népôs de la Nouvelle-Guinée ${ }^{33}$ » de Charles Mensuel, qui reprend en trois pages le voyage du marquis, accompagné pour la première fois de trois illustrations. Si dans La Science Française les considérations géographiques prennent le pas sur l'anecdotique, l'article de Charles Mensuel inverse la tendance en se concentrant sur la figure du sorcier néo-guinéen, le népô, dont l'emprise sur ses congénères est dénoncée par l'auteur. À nouveau, les notes de Cacqueray de Lorme sont évoquées comme sources :

Un explorateur, qui a beaucoup voyagé, a bien voulu me confier ses notes relatives à un voyage qu'il a fait en 1895, dans la Nouvelle-Guinée anglaise, notes d'autant plus précieuses que la région parcourue par mon ami, M. de Cacqueray de Lorme, était ignorée presque complètement ; personne ne s'y était encore aventuré hormis M. d'Albertis qui n'a que très imparfaitement exploré le littoral [... $]^{34}$.

Ces premières lignes ne sont pas sans rappeler l'article de Gaston Bertrand ; pourtant, le ton est différent et dès le début, Charles Mensuel qualifie les Néo-Guinéens de "peuplades bizarres ». La première page est consacrée à deux anecdotes retraçant les mauvaises aventures d'un père du Sacré-Cœur avec le sorcier du village de Bihoto : ce dernier, après avoir tenté d'assassiner le père, empoisonna son cheval. Mais le reste de l'article est encore plus surprenant: Mensuel détaille l'assassinat d'un sorcier néoguinéen par Cacqueray de Lorme, et cite directement les paroles de l'explorateur :

«Il ne m'a été donné qu'une fois d'avoir affaire à un népô, et je suis obligé de confesser que la fin de l'aventure lui a enlevé tout moyen d'action. » [...] M. de Cacqueray était séparé du vieux brigand par une distance de 50 à 60 mètres ; aussi n'hésita-t-il pas un seul instant à lui envoyer une balle. [...] Courir sur lui, le débarrasser de ses plumes et de ses gris-gris qu'il voulait rapporter comme dépouilles opimes, fut un jeu pour l'explorateur qui, aidé par son Malais, prit le corps du népô et le jeta dans le fleuve, où se montraient plusieurs têtes de crocodiles qui avaient surgi tout doucement des fouillis des lianes de la rive opposée, en quête d'un bon repas ${ }^{35}$.

Le danger de l'aventure - duquel découle le succès de l'aventurier - s'incarne dans les détails: après le meurtre, les crocodiles sont prêts à faire disparaître le corps du sorcier ; un frisson d'horreur parcourt le lecteur. 
Fig. 4

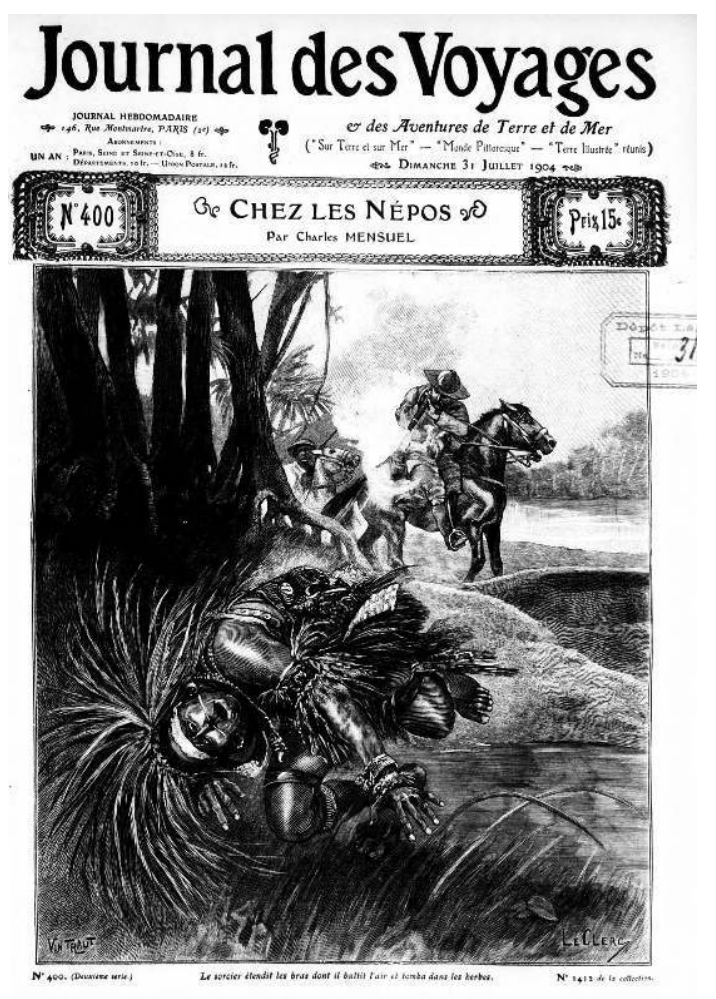

Journal des Voyages et des Aventures de Terre et de Mer, n 400, 31 juillet 1904, p. 145.

Quelle est la part de vérité dans ce récit? Il n'est pas improbable que Cacqueray de Lorme ait effectivement tué un sorcier: la violence a malheureusement souvent été l'issue aux rencontres entre les explorateurs et colons occidentaux, et les populations autochtones. Quoi qu'il en soit, cette violence s'affiche en une de l'hebdomadaire : le meurtre prend un air d'aventure, la légende précise que «Le sorcier étendit les bras dont il battit l'air et tomba dans les herbes ${ }^{36}$ » (fig. 4). Le Journal des Voyages, qui existe depuis 1877, est devenu spécialiste de ces unes tragiquement violentes; la revue était réputée pour mêler des récits sérieux d'explorateurs aux histoires les plus extravagantes d'aventuriers intrépides. Ici, la une cherche à attirer un lecteur avide d'aventures en terres lointaines en se concentrant sur une partie de la narration de Charles Mensuel, la plus racoleuse. Si cette illustration sort tout droit de l'imaginaire de son auteur, un certain Leclerc, les deux autres illustrations de l'article reprennent des photographies de Cacqueray de Lorme. Un groupe de danseurs apparaît légèrement modifié (fig. 5): la légende indique qu'il s'agit de musiciens du village de Rahi-Rahi, dont le texte nous informe qu'il se situe sur le haut Saint-Joseph ${ }^{37}$. Sur cette gravure, la colonne gauche du groupe de danseurs a été effacée, la rue et la construction missionnaire en arrière-plan ont été remplacées par une végétation esquissée mais luxuriante. La troisième illustration de l'article est l'exacte reproduction d'une vue de Cacqueray, un portrait d'un groupe sur la plate-forme d'un bâtiment: elle est ici légendée "Chefs et femmes papous devant la principale case du village de Bihoto ». Une carte publiée par les missionnaires du Sacré-Cœur fait mention de ce village, orthographié "Bioto ", situé tout près de la côte : les parures de coquillages portées par les hommes, caractéristiques des populations du Golfe de Papouasie, attestent de la situation côtière du village. Si Charles Mensuel ancre son récit dans les deux villages de 
Bihoto et de Rahi-Rahi, il n'évoque pas les musiciens du premier ni la «principale case » du second. Aucun lien direct n'existe entre ces deux illustrations et le déroulement de la narration si ce n'est la localisation assurée par les légendes; la présence de ces images permet la projection du lecteur dans un espace réel.

Fig. 5
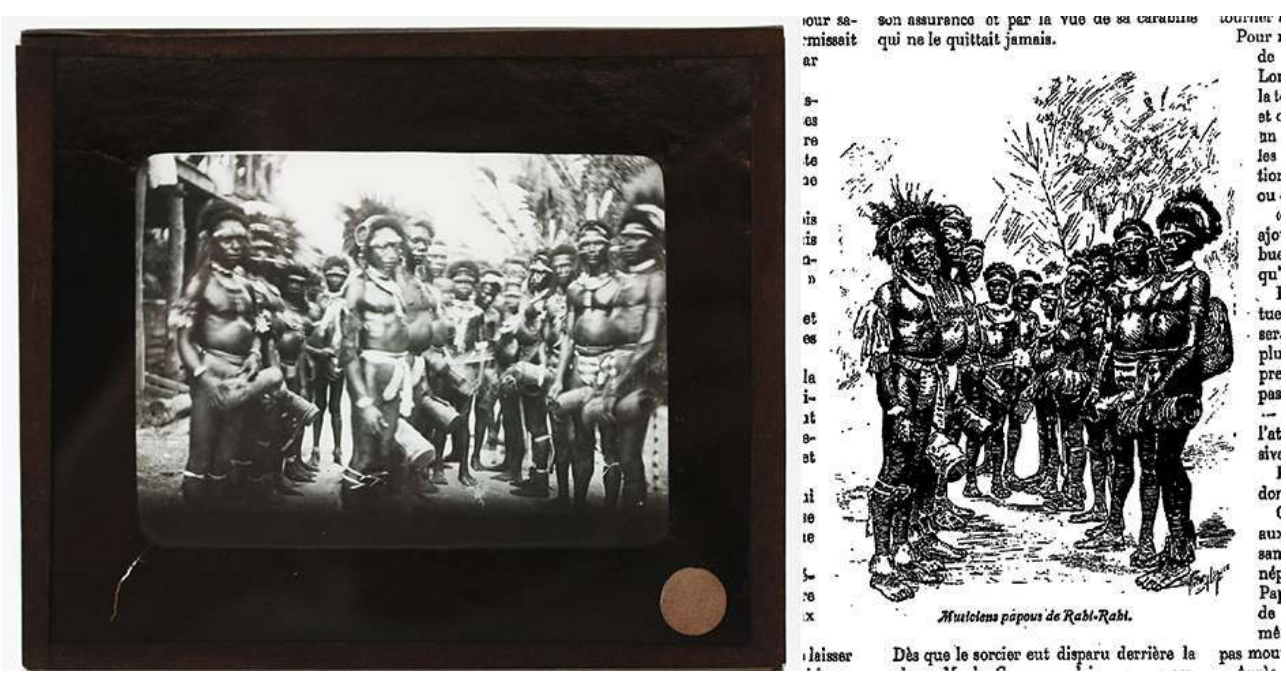

Charles Auguste de Cacqueray de Lorme, Nouvelle-Guinée, PV0045252, plaque provenant de la collection de plaques de projection du Musée de l'Homme (c) musée du quai Branly - Jacques Chirac, dist. RMN-Grand Palais / image musée du quai Branly - Jacques Chirac (gauche) et Musiciens papous de Rahi-Rahi (droite) d'après Charles Mensuel, "Les Népôs de la Nouvelle-Guinée », Le Journal des Voyages et des Aventures de Terre et de Mer, n’ 400, 31 juillet 1904, p. 148.

\section{Emprunts et détournements}

16 Cette publication de 1904 par Charles Mensuel est la première à réutiliser des photographies de Cacqueray de Lorme, mais ces dernières sont exemptes de tout crédit à l'exception de celui du graveur qui signe la transposition du groupe de danseurs. Six années plus tard, le marquis lui-même prend enfin la plume pour raconter son expédition dans un long article d'une douzaine de pages publié dans Le Tour du Monde ${ }^{38}$. Fait notable, le récit est accompagné de pas moins de quatorze reproductions photographiques. Ces illustrations sont légendées avec des références explicites au voyage du marquis. Ainsi, le lecteur rencontre un " groupe de femmes nous apportant des vivres », observe un portrait d'un «type d'homme du village de Beïpaa » et assiste à une «fête du Tadzou, en l'honneur de notre présence à Mékéo » (fig. 6) ; certaines de ces légendes renvoient directement le lecteur à la page où se trouve l'épisode illustré par l'image en question. Pourtant, le soin particulier apporté à ces photographies tranche avec les défauts techniques des neuf plaques photographiques de facture amateure du marquis; les reproductions sont bien entendu exemptes de tout crédit. Pour résoudre cette discordance, il faut regarder du côté des publications allemandes : onze de ces photographies proviennent ainsi d'un album de 1894, Album von Papúa Typen, Neu Guinea und Bismark Archipel ${ }^{39}$, considéré par les historiens de la PapouasieNouvelle-Guinée comme l'ouvrage illustré de référence de la fin du $\mathrm{XIX}^{\mathrm{e}}$ siècle $^{40}$. Outre l'emprunt non crédité des photographies de Richard Parkinson, les images sélectionnées pour l'article du Tour $d u$ Monde ont toutes été réalisées en Nouvelle 
Bretagne et non à Mékéo, Pinoukapa ou en pays Roro, comme l'indiquent les légendes françaises. Les "Pirogues en fête près de Pinoupaka» et la "Fête ou Tadzou, en l'honneur de notre présence à Mékéo » (fig. 6) sont en réalité deux « Dukduk Ahnenfest in Neu Pommern ${ }^{41}$ ». Le «Type d'homme du village de Beïpaa » (fig. 6) est un « Mann von Neu Pommern (Neu Britannien)» (fig. 7).

Fig. 6

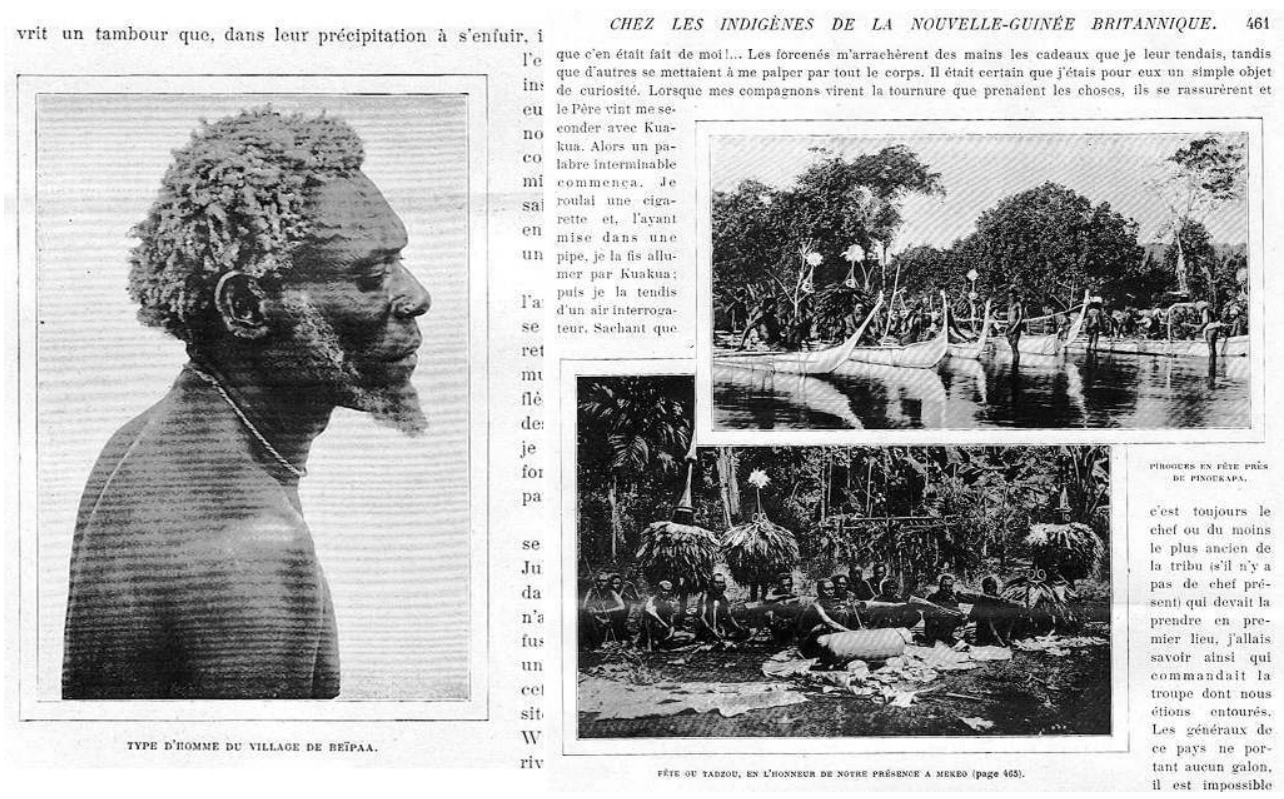

Type d'homme du village de Beïpaa et Pirogues en fête près de Pinoukapa ; Fête du Tadzou, en l'honneur de notre présence à Mékéo (page 465) d'après Marquis de Cacqueray, "Chez les indigènes de la NouvelleGuinée britannique », Le Tour du Monde : nouveau journal des voyages, tome 16, n 39, 24 septembre 1910, p. 460-461 
Fig. 7

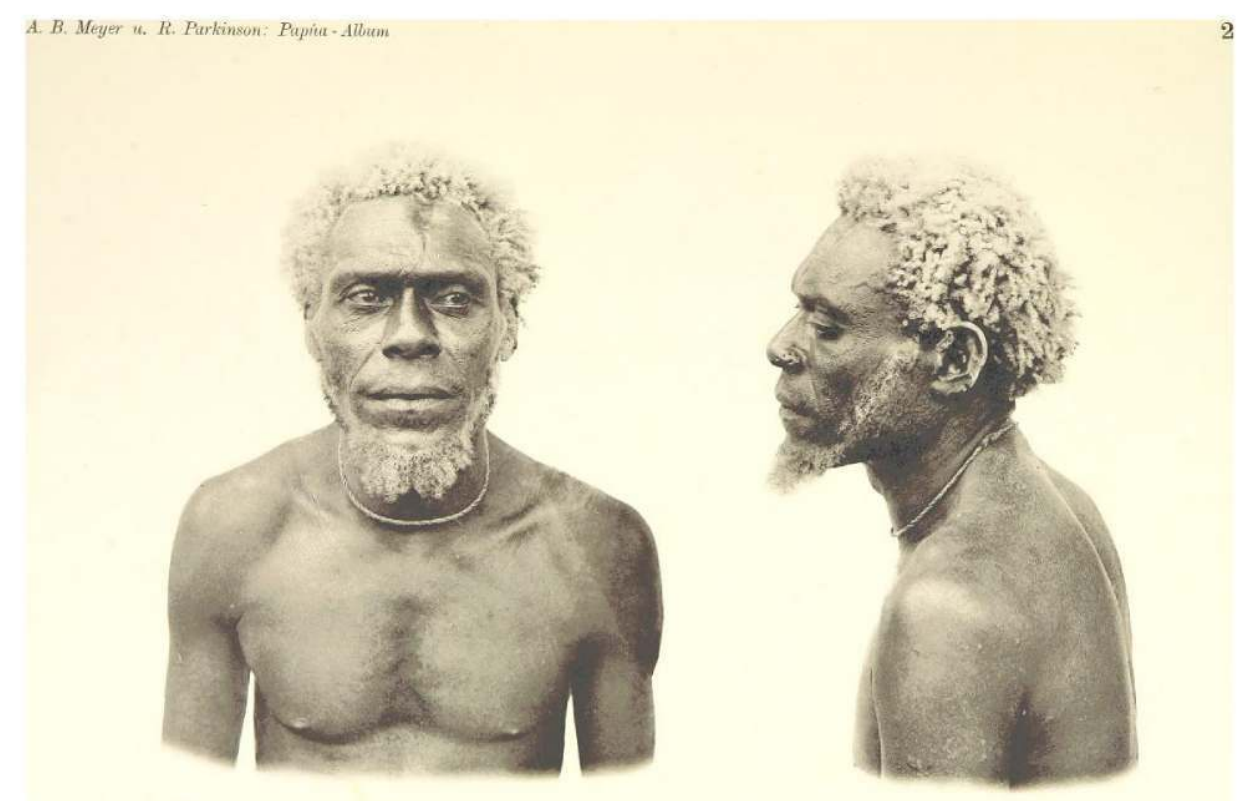

Mann von Neu Pommern (Neu Britannien)

Richard Parkinson, Mann von Neu Pommern (Neu Britannien), d'après Adolf Meyer Bernhard, Richard Parkinson, Album von Papúa Typen, Neu Guinea und Bismark Archipel, 1894, p. 47.

Cette réutilisation montre le peu de cas qui est fait, par les auteurs et les éditeurs, de la spécificité des différentes régions néo-guinéennes et des îles environnantes. Le sens initial des photographies est totalement évacué. Réalisées dans le cadre de l'étude des populations et de la culture matérielle de Nouvelle-Bretagne, les photographies de Parkinson endossent le rôle de certificats d'authenticité du récit de l'explorateur français qui se déroule dans une tout autre région géographique. Cet emploi frauduleux de photographies détournées remet en cause la crédibilité des récits de Cacqueray de Lorme. Les photographies aux légendes inventées abusent le lecteur qui avait pu voir en elles un témoignage privilégié de l'aventure du marquis. Les légendes ont un rôle d'autant plus important dans les photographies de Nouvelle-Guinée que les lecteurs auxquels elles s'adressent sont généralement peu informés sur cette île lointaine et n'ont donc pas d'outil de comparaison ou de vérification de l'information apportée. Mais pour le marquis de Cacqueray de Lorme, comme pour les éditeurs du Tour $d u$ Monde, peu importe l'authenticité de la légende, et donc du lien supposément fondé entre texte et image : ils produisent un récit exotique illustré d'images appartenant à la sphère du «lointain » dans l'imaginaire collectif du début du siècle. L'auteur et/ou l'éditeur se saisissent des caractéristiques prétendument objectives du médium photographique pour les plier à leurs intentions peu scrupuleuses. 


\section{La scientificité en recul face aux impératifs commerciaux}

18

Les éditeurs du Journal des voyages et du Tour du Monde poursuivent un but commercial ; leurs revues sont d'ailleurs concurrentes - il est amusant de constater que toutes deux publient sur l'expédition de Cacqueray de Lorme, mais en ne mobilisant pas les mêmes moyens. La simplicité du propos anthropologique du marquis n'est pas due uniquement à son manque de scientificité ou au contexte français au sein duquel l'anthropologie est encore une science balbutiante ; en effet, la logique commerciale de ces revues s'oppose à la logique scientifique et cette confrontation ne peut aboutir qu'à la distorsion et à la réduction du propos.

L'article du Tour du monde, Cacqueray de Lorme le conclut de la façon suivante :

Bien que je sois resté plusieurs mois au milieu des indigènes de Nouvelle-Guinée et que chaque jour amenât pour moi son contingent d'observations, je ne m'étendrai pas plus longtemps sur des scènes ou des aventures dont j'ai choisi les plus caractéristiques pour les présenter au lecteur. Elles me paraissent suffisantes pour donner une idée des mœurs et de la mentalité qu'on trouve chez ces peuplades, auxquelles on peut donner encore avec exactitude l'épithète de sauvage et qui habitent une colonie anglaise dont le territoire, sauf sur les côtes, est de nos jours si peu connu. [...] Malgré tout, le Territory of Papua ne sera jamais un des plus beaux fleurons de la couronne coloniale de la Grande-Bretagne ${ }^{42}$.

Comme nous l'avons vu, ce ton particulièrement méprisant est déjà présent lors des interventions du marquis à la Société de géographie en 1896 puis d'Anthropologie en $1901^{43}$. Ces observations naissent et alimentent le terreau des conceptions racialistes qui classent les peuples mélanésiens parmi les « races inférieures ». Comment peut-on alors avancer que ces conférences, ou bien ces articles, constituent un lieu de rencontre avec l'Autre et l'Ailleurs? Les neuf plaques de projection de Cacqueray de Lorme participent de la dynamique relayant les clichés raciaux et alimentant la construction d'un imaginaire collectif fantasmé qui, comme on l'a vu à travers les récits du marquis, associent souvent le sensationnalisme à l'île de Nouvelle-Guinée.

\section{Conclusion}

Le personnage de Cacqueray de Lorme et sa production visuelle et littéraire en relation avec la Nouvelle-Guinée rendent admirablement compte des lignes de force qui soustendent le développement concomitant des expéditions d'exploration d'initiative privée, de la science anthropologique et des théories racialistes, ainsi que celui de la presse illustrée en ce début de $\mathrm{xx}^{\mathrm{e}}$ siècle. La courte histoire que nous raconte le parcours du marquis est un exemple parmi tant d'autres de la constitution et de la sédimentation de l'imaginaire collectif occidental.

21 Appartenant à la classe bourgeoise française, Cacqueray de Lorme organise seul cette expédition en Nouvelle-Guinée : il n'évolue pas dans les sphères du pouvoir et ne se rapproche des sociétés savantes qu'au moment de son séjour. Il incarne à merveille ce cliché de l'aventurier indépendant dont le courage sera justement mis en avant dans les publications à visée commerciale. Nous ne connaissons pas les motifs qui le poussent à effectuer cette traversée du globe pour se rendre sur l'île mais nous ne pouvons sousestimer le mystère qui entoure cette région dont Jules Girard disait qu'elle était «encore la région la moins connue du globe ${ }^{44}$ ». En outre, toute une mythologie 
s'élabore autour des populations néo-guinéennes au travers de publications de récits de voyage, notamment britanniques, qui mettent en avant le cannibalisme comme pratique régulière sur ces territoires ${ }^{45}$. On peut alors penser qu'avant de partir, Cacqueray avait déjà une idée de la Nouvelle-Guinée et de ses habitants. Que se passe-til s'il ne trouve pas ce qu'il est venu chercher? Il suffit de le fabriquer : fabriquer ces terres lointaines en proie à l'obscurantisme des populations sauvages auquel viendra bien évidemment s'opposer la raison occidentale supportée par le progrès technique et scientifique.

Le médium photographique s'inscrit à ce moment-là, et particulièrement dans les cercles des sociétés savantes, comme une preuve incontestable, soutien du propos développé. Mais le discours de Cacqueray de Lorme se situe à un double niveau : s'il s'énonce dans la sphère scientifique, où il relaie des idées déjà professées avant lui, il se déplace également du côté du récit populaire, grâce à ce nouveau médium qu'est la presse illustrée, fournissant danger, rebondissements et violence à des lecteurs avides d'exotisme en abritant son récit sous l'autorité du médium photographique. Le cheminement que nous venons d'effectuer met ainsi clairement en relief les questionnements intrinsèquement liés à l'étude de l'image photographique et l'importance de la recherche sur les conditions de production et d'utilisation de ces images, tour à tour témoins des premières années de l'installation missionnaire catholique en Nouvelle-Guinée et vectrices des manipulations du marquis français.

\section{NOTES}

1. Lettre du 25 mars 1881, du cardinal Siméoni au père Jules Chevalier, dans André Dupeyrat, Papouasie, Histoire de la Mission (1885-1935), Paris, Éditions Dillen, 1935, p.31. Notre source principale sur l'établissement de la mission sur la côte sud-est est André Dupeyrat, missionnaire et historien de la Société. Les archives des missionnaires d'Issoudun semblent avoir disparu en Nouvelle-Guinée; cette information nous a été transmise par le père Philippe Séveau, ancien missionnaire en Papouasie-Nouvelle-Guinée, lors d'un entretien mené à la maison des Missionnaires du Sacré-Cœur de Paris, le 20 décembre 2014.

2. Jules Chevalier naît en 1824 et manifeste très tôt une vocation religieuse. Ordonné en 1851, il est nommé à Issoudun trois ans plus tard où il créera la Société des Missionnaires du Sacré-Cœur d'Issoudun.

3. Cf. l'étude menée par Amandine Venard sur le fonds photographique des CEuvres pontificales missionnaires, montrant que la majorité de la production photographique se concentre entre les années 1930 et 1940 : Amandine Venard, Regards de missionnaires catholiques sur un paradis perdu, 1890-1950, photographies en provenance de Papouasie-Nouvelle-Guinée, sous la direction de Philippe Delisle, Université Jean Moulin, Lyon III, 2014, p. 74. Notons tout de même que le musée du quai Branly - Jacques Chirac a récemment acquis un ensemble de plus d'une centaine de photographies de l'installation missionnaire catholique en Nouvelle-Bretagne, au nord-est de l'île de Nouvelle-Guinée, ensemble daté des années 1890.

4. Cf. tables décennales de Presles-sur-Boves, 1853-1862, site Internet des Archives départementales de l'Aisne. 
5. Bulletin de la Société de Géographie, tome II, $2^{\mathrm{e}}$ semestre, 1900.

6. Cf. Gaston Bertrand, "Pérégrinations d'un compatriote dans la Papouasie inconnue ", La Science Française, 5 octobre 1900, n 297, p. 116-117. Les Bulletins de la Société d'anthropologie de Paris indiquent que le marquis serait toujours en Nouvelle-Guinée au mois de juin 1895, cf. «Photographies et objets de la Nouvelle Guinée anglaise », Bulletins de la Société d'anthropologie de Paris, Ve Série. Tome 2, 1901, p. 393-398.

7. «Et comme le voyageur avait, sur la fin de sa relation, exprimé ses regrets de l'interruption que son voyage avait dû subir, le Président lui dit : "Je ne saurais trop vous engager à reprendre une exploration si malheureusement interrompue et à la pousser jusqu'à ses dernières limites. Espérons que cette fois vous pourrez rapporter les documents que vous aurez recueillis et en faire profiter la science." " dans Compte rendu des séances de la Société de géographie et de la Commission centrale, 19 juin 1896, p. 268.

8. Gaston Bertrand, op. cit. note 6.

9. Avis de décès Charles Auguste Honoré Cacqueray de Lorme, Base des Morts pour la France de la Première Guerre mondiale, ministère de la Défense.

10. André Jullien naît à Marseille en 1861 ; il est ordonné prêtre en 1886. Il débarque à Yule Island le 22 février 1894 et occupe la station de Tsiria. Il tient le poste de supérieur de la mission de 1895 à 1909. Il rentre en Europe en 1911 où il est nommé à Rome. Il décédera à Marseille, le 15 décembre 1920. Cf. Diane Langmore, Missionary Lives, Papua, 1874-1914, Honolulu, University of Hawaii Press, 1989, p. 306.

11. Victor de Rijcke naît en Belgique en 1871. Il arrive en Nouvelle-Guinée en 1894 comme scolastique, se fait ordonner prêtre le 21 décembre 1895 et occupe la station de Vanamai. Il meurt le 5 janvier 1899, sur l'île de Yule. Cf. Diane Langmore, op. cit. note 10, p. 304.

12. La Presse, Paris, 18 juin 1896.

13. Annonce publicitaire de A. Molteni in Revue géographique internationale, 1885, n. p.

14. Olivier Loiseaux (dir.), «Le fonds photographique de la Société de géographie ", Trésors photographiques de la Société de géographie, Paris, Bibliothèque nationale de France, Glénat, 2006.

15. Bertrand Lavedrine, (Re)Connaître et conserver les photographies anciennes, Paris, Éditions du Comité des travaux historiques et scientifiques, 2008, p. 68.

16. «Photographies et objets de la Nouvelle Guinée anglaise », op. cit. note 6, p. 396-397.

17. «M. le marquis de Cacqueray de Lorme [...] croit que les Papouas sont le résultat d'un mélange d'indiens de l'Amérique (aux cheveux lisses) avec des nègres aux cheveux crépus [...]. », dans «Photographies et objets de la Nouvelle Guinée anglaise », op. cit. note 6, p. 394.

18. «Photographies et objets de la Nouvelle Guinée anglaise », op. cit. note 6, p. 395-396.

19. Le parcours des plaques de projection met en lumière les connexions entre ces différentes institutions : Paul Rivet, titulaire de la chaire d'anthropologie du Muséum, est aussi le directeur du Musée d'Ethnographie du Trocadéro entre 1929 et 1937, musée qui deviendra le Musée de l'Homme en 1938. Cf. Claude Blanckaert (dir.), Le Musée de l'Homme : histoire d'un musée laboratoire, Paris, Muséum national d'histoire naturelle / Éditions Artlys, 2015.

20. André Jullien, Album des missions de la Nouvelle-Guinée confiées à la Société des missionnaires du Sacré-Cœur, Paris, imprimerie D. Dumoulin et Cie, 1898.

21. «Photographies et objets de la Nouvelle Guinée anglaise », op. cit. note 6, p. 395.

22. Jules Girard, «Les connaissances actuelles sur la Nouvelle-Guinée », dans Bulletin de la Société de Géographie, $6^{\mathrm{e}}$ série, $\mathrm{n}^{\circ} 4,1872$, p. 465.

23. Le Journal des Voyages et des Aventures de terre et de mer, juillet-décembre 1896, p. 191.

24. Revue de géographie, tome XXXIX, juillet-décembre 1896, p.73-74; Revue de géographie, tome XL, janvier-juin 1897, p. 285-286.

25. Le Journal des Voyages et des Aventures de terre et de mer, op. cit. note 23.

26. Revue de géographie, tome XXXIX, juillet-décembre 1896, p. 73-74. 
27. Gaston Bertrand, op. cit. note 6.

28. « Ici, une note de l'explorateur, concernant les procédés agronomes des indigènes. [...] nous sommes dans une région sur laquelle $\mathrm{M}$. de Cacqueray de Lorme a été le premier à nous donner des notes [...]. » dans Gaston Bertrand, op. cit. note 6, p. 117.

29. Idem, Ibidem, p. 116.

30. Id., Ibid.

31. Id., Ibid.

32. Id., Ibid.

33. Charles Mensuel, «Les Népôs de la Nouvelle-Guinée », Le Journal des Voyages et des Aventures de Terre et de Mer, $\mathrm{n}^{\circ}$ 400, 31 juillet 1904, p. 146-148.

34. Idem, Ibidem, p. 146.

35. Id., Ibid., p. 147.

36. Id., Ibid., p. 145.

37. Aucune mention de ce village n'a été retrouvée dans les sources d'époque.

38. Cacqueray de Lorme, "Chez les indigènes de la Nouvelle-Guinée britannique ", Le Tour du Monde : nouveau journal des voyages, tome 16, $\mathrm{n}^{\circ}$ 39, 24 septembre 1910, p. 457-468.

39. Adolf Bernhard Meyer, Richard Parkinson, Album von Papúa Typen, Neu Guinea und Bismark Archipel, Dresde, Verlag von Stengel und Markert, 1894.

40. Max Quanchi, «Photography and History in the Pacific Islands. Visual Histories and Photographic Evidence », The Journal of Pacific History, vol. 41, n² 2, septembre 2006, p. 168.

41. Adolf Bernhard Meyer, Richard Parkinson, op. cit. note 39, p. 53.

42. Cacqueray de Lorme, op. cit. note 38, p. 467-468.

43. «Les Papous vivent dans l'état sauvage le plus complet [...]. Ils sont peu intelligents et d'une nature très paresseuse. On aura beaucoup de peine à les civiliser ; [...]. Au milieu de ces indigènes on se croirait revenu à l'âge de pierre " dans Compte rendu des séances de la Société de géographie, op. cit. note 7, p. 267.

44. Jules Girard, op. cit. note 22, p. 450.

45. Cf. Hugh Romilly, The Western Pacific and New Guinea: notes on the natives, Christian and cannibal, with some account of the old labour trade, Londres, J. Murray, 1886 ; Samuel Macfarlane, Among the cannibals of New Guinea: being the story of the New Guinea mission of the London Missionary Society, Londres, J. Snow, 1888 ; Herbert Cayley-Webster, Through New Guinea and the Cannibal Countries, Londres, T. Fisher Unwin Paternoster Square, 1898.

\section{RÉSUMÉS}

Il est amusant de constater que le détournement des images, celui qui nous est devenu si évident et omniprésent dans notre quotidien, a une histoire longue ; on le retrouve dès le début du $\mathrm{xx}^{\mathrm{e}}$ siècle lorsque l'on s'intéresse aux publications de la presse illustrée française relatant les récits d'un explorateur des terres de Nouvelle-Guinée. C'est l'histoire du marquis de Cacqueray de Lorme, héritier bourgeois, qui part dans l'année 1895 retrouver les quelques missionnaires catholiques français présents en Nouvelle-Guinée, cette immense île encore majoritairement inconnue des Occidentaux. 
It is amusing to observe that the misappropriation of images, which has become so obvious and omnipresent in our daily lives, has a long history; it may be found from early twentieth century when one takes a look at the publications of the French illustrated press recounting the narratives of an explorer of New Guinea. It is the story of Marquis de Cacqueray de Lorme, a bourgeois heir, who set off in 1895 to find the few French Catholic missionaries in New Guinea, the vast island largely unknown to Westerners.

\section{INDEX}

Mots-clés : photographie, Océanie, Pacifique, Nouvelle-Guinée, colonisation, évangélisation, exotisme, culture visuelle, imaginaire collectif

Keywords : photography, Oceania, Pacific, New Guinea, colonisation, evangelisation, exoticism, visual culture, collective imagination

\section{AUTEUR}

\section{VICTOIRE LALLOUETTE}

Diplômée d'un master de l'Université Paris 1 Panthéon-Sorbonne (recherche en histoire de l'art, spécialité histoire de la photographie) et du deuxième cycle de l'École du Louvre (parcours « recherche » en histoire de l'art appliquée aux collections), Victoire Lallouette a consacré ses travaux de recherche à l'usage du médium photographique dans le Pacifique sud durant le moment colonial. Sous la direction de Dominique de Font-Réaulx et de Michel Poivert, elle a étudié les collections photographiques du musée du quai Branly - Jacques Chirac, d'abord en Polynésie française, puis en Papouasie-Nouvelle-Guinée à la jonction des XIX et $\mathrm{XX}^{\mathrm{e}}$ siècles. ***

The holder of a Master from Université Paris 1 Panthéon-Sorbonne (art history, specialising in the history of photography) and of a postgraduate degree from the École du Louvre (art history research applied to collections), Victoire Lallouette has focussed her research on the use of the photographic medium in the South Pacific during the colonial period. Supervised by Dominique de Font-Réaulx and Michel Poivert, she studied the photographic collections of the Musée du Quai Branly - Jacques Chirac, initially focussing on French Polynesia, then on Papua-New Guinea at the turn of the twentieth century. 